\title{
EL DESEMPEÑO RECIENTE DEL SECTOR AGRARIO PAMPEANO: SUS POTENCIALIDADES Y PERSPECTIVAS. ANÁLISIS DE LAS EXPLICACIONES ECONÓMICAS
}

\section{THE RECENT PERFORMANCE OF THE AGRICULTURAL SECTOR OF THE PAMPAS: THEIR POTENTIAL AND PROSPECTS. ANALYSIS OF ECONOMIC EXPLANATIONS}

\section{RESUMEN}

\author{
Estefanía Soledad Dileo \\ FCE - Universidad de Buenos Aires \\ Ciudad de Buenos Aires, Argentina \\ dileoestefania@gmail.com
}

La inserción de Argentina como país exportador de productos primarios y manufacturas de origen agrario explica la importancia de la producción agrícola en el proceso de acumulación nacional. En línea con esto, la ciencia económica, desde distintas concepciones, se ha abocado al estudio de dicho sector para conocer sus potencialidades para impulsar el crecimiento y el desarrollo de la economía argentina. Como resultado de esto, al aproximarse a la literatura especializada, se encuentra una multiplicidad de enfoques económicos que explican y analizan el sector agrario pampeano desde distintas perspectivas.

Desde nuestro punto de vista, el disenso que se verifica en la literatura sobre el sector, guarda relación con la forma en la cual la ciencia económica aborda los fenómenos que constituyen su objeto de estudio. La propuesta del presente trabajo consiste en realizar un análisis documental de las distintas explicaciones sobre el desempeño reciente del sector agrario pampeano -específicamente, el boom sojero-, estructurándolo de tal forma de poner de manifiesto la manera en que abordan lo que pretenden explicar. El resultado del análisis no sólo logra poner de relieve la correspondencia entre las formas de conocer de las explicaciones agrarias y los postulados epistemológicos de la ciencia económica, sino que también aquello en lo que se sustenta la falta de consenso entre los especialistas para explicar el sector agrario pampeano.

PALABRAS CLAVE: Desarrollo Económico; Sector Agrario Pampeano; Boom Sojero; Análisis Bibliográfico.

\section{ABSTRACT}

The insertion of Argentina as an exporter of primary products and manufactures of agricultural origin explains the importance of agricultural production in the process of national accumulation. In consequence of this, economic science, from different conceptions, has focused on the study of this sector to know its potential to boost the growth and development

"Visión de Futuro" Año 16, Volumen No 23 Nº1, Enero - Junio 2019 - Pág. 42 - 59

URL de la Revista: http://revistacientifica.fce.unam.edu.ar/

URL del Documento: http://revistacientifica.fce.unam.edu.ar/index.php?option=com content\&view=article\&id=492\&ltemid=101

ISSN 1668 - 8708 - Versión en Línea

E-mail: revistacientifica@fce.unam.edu.ar 
of the Argentine economy. As a result of this, when approaching the specialized literature, there is a multiplicity of economic approaches that explain and analyze the Pampean agricultural sector from different perspectives.

From our point of view, the dissensus that is verified in the literature on the sector, is related to the way in which economic science addresses the phenomena that constitute its object of study. The aim of the present work consists in making a documentary analysis of the different explanations about the recent performance of the Pampean agricultural sector specifically, the soy boom-, structuring it in such a way to expose the way in which they approach what they intend to explain. The result of the analysis not only manages to highlight the correspondence between the ways of knowing about agrarian explanations and the epistemological postulates of economic science, but also that which underpins the lack of consensus among specialists to explain the sector agrarian pampeano.

KEY WORDS: Economic Development; Pampeano Agrarian Sector; Soybean Boom; Bibliographical Analysis.

\section{INTRODUCCIÓN}

La participación de Argentina en el comercio internacional como uno de los mayores exportadores de productos primarios y manufacturas de origen agrario determina la importancia de la producción agrícola pampeana en el proceso de acumulación nacional. Así, con el interés puesto fundamentalmente en la comprensión de las potencialidades de la producción agraria de la Pampa Húmeda para impulsar el crecimiento y el desarrollo económico nacional, aparece, desde la teoría económica, la necesidad de estudiar a este sector. No obstante, una primera aproximación a la bibliografía especializada pone al descubierto que existen diferentes concepciones acerca del desempeño y las características de la producción agraria pampeana.

Específicamente, esta particularidad se hace notoria en dos momentos históricos determinados. En primer lugar, en las décadas de 1960 y 1970 cuando, luego de un prolongado período de 'estancamiento' de la producción agraria pampeana -desde la década de 1930 hasta la década de 1970-, se desarrolla un importante debate entre los estudiosos del sector con el objeto de dilucidar las causas de su deterioro, sin llegar a un consenso, lo cual ha sido reconocido por numerosos análisis que retoman dicha discusión (Sábato, 1987; Barsky y Gelman, 2001). En segundo lugar, años después, desde mediados de la década de 1990, pero más profundamente durante la primera década del nuevo siglo, cuando el desempeño de la producción de granos vuelve a destacarse, aunque esta vez por su "Visión de Futuro" Año 16, Volumen No 23 N¹, Enero - Junio 2019 - Pág. 42 - 59 URL de la Revista: http://revistacientifica.fce.unam.edu.ar/

URL del Documento: http://revistacientifica.fce.unam.edu.ar/index.php?option=com content\&view=article\&id=492\&ltemid=101 ISSN 1668 - 8708 - Versión en Línea

E-mail: revistacientifica@fce.unam.edu.ar 
espectacular crecimiento. Nuevamente, a la hora de explicar este hecho, pareciera que no hay consenso entre los autores de la literatura especializada.

Desde nuestra perspectiva, el disenso que se verifica en la literatura sobre el sector agrario pampeano motiva a estudiar las distintas concepciones, prestando especial atención a la forma en la cual cada una de ellas aborda el fenómeno que se pretende explicar. Así, nuestro principal objetivo consiste en echar luz sobre las potencialidades y limitaciones de los enfoques económicos convencionales sobre el sector agrario pampeano para dar cuenta de sus características y su desempeño reciente, a partir de una mirada crítica respecto del método de conocimiento en el cual se sustentan.

A nuestro entender, la falta de consenso en la literatura especializada se basa, fundamentalmente, en que las distintas posturas, al analizar y caracterizar el desempeño del sector, construyen su explicación a partir de la identificación de un motor explicativo específico, de acuerdo con el método general de conocimiento de la ciencia económica. En otras palabras, cada concepción, ya sea para explicar un período de estancamiento o de auge de la producción agraria pampeana, luego de evaluar, implícita o explícitamente, el conjunto de determinaciones que caracterizan el comportamiento del sector durante un período dado, realiza hipótesis con el objetivo de construir la explicación. En pos de dicha construcción, mientras algunas características tendrán un rol preponderante, otras, en cambio, serán secundarias o incluso consecuencia de las primeras. En este sentido, decimos que cada concepción hace un determinado recorte de la realidad -al seleccionar lo que considera relevante para la explicación que pretende construirse-, para luego hipotetizar sobre el comportamiento de aquellos elementos seleccionados y construir así la explicación que dé cuenta del fenómeno abordado. El resultado de esto es una multiplicidad de enfoques, que se presentan como igualmente válidos (Denari, 1991). Como cuestión problemática adicional, lo que suele encontrarse es que cada explicación es temporal; esto es, se desarrolla para un momento histórico específico sin pretensión de dar cuenta acerca del desenvolvimiento del sector en el mediano o largo plazo.

En el presente trabajo nos centraremos en las concepciones que analizan el desempeño de la producción agraria pampeana desde mediados de la década de 1990 hasta fines de la primera década del nuevo siglo-específicamente, el boom de la producción sojera- con el objetivo de aportar a la comprensión de las potencialidades y perspectivas del sector en la actualidad. En línea con esto, constituye un primer objetivo específico de esta investigación verificar si efectivamente se produce nuevamente una época de disenso entre los estudiosos de la producción agraria pampeana, en relación al desempeño reciente del sector, debido a que este caso -a diferencia del debate en los años setenta- no ha sido extensamente analizado por la literatura. En segundo lugar, nos proponemos identificar en las distintas

\footnotetext{
"Visión de Futuro" Año 16, Volumen No 23 N¹, Enero - Junio 2019 - Pág. 42 - 59

URL de la Revista: http://revistacientifica.fce.unam.edu.ar/

URL del Documento: http://revistacientifica.fce.unam.edu.ar/index.php?option=com content\&view=article\&id=492\&ltemid=101 
concepciones analizadas el principal factor explicativo esgrimido para dar cuenta del desempeño y las características del sector; esto es, señalar el aspecto del mismo en el cual hacen hincapié, la parcialidad con la que lo abordan.

Entendemos que la importancia de estudiar la literatura especializada para identificar estas cuestiones radica en la necesidad de poner de manifiesto el imperativo de avanzar en la comprensión del sector, en su unidad y especificidad. Así, el objetivo general en el cual se enmarca esta investigación, aunque exceda los límites del presente trabajo, consiste en mostrar la necesidad de una mirada complementaria acerca del desempeño reciente y las características de la producción agraria pampeana para comprenderla en la unidad de la acumulación de capital en Argentina y su papel en la División Internacional del Trabajo (DIT). Del mismo modo, la reflexión crítica sobre el desempeño reciente del sector, permite contar con mayores herramientas a la hora de planificar estrategias de producción en el agro pampeano.

\section{Metodología}

En el presente trabajo nos centramos en la producción agraria pampeana, dejando de lado no sólo la actividad ganadera sino también los cultivos de otras regiones del país. Esta decisión se basa, no sólo en la mayor literatura disponible para esa producción, sino también en su mayor relevancia para la economía nacional.

El abordaje metodológico es de orden cualitativo, utilizando el método documental. La empiria está entonces constituida por el trabajo objetivado de los investigadores, esto es, la producción científica. Por lo tanto, las fuentes son textos, bajo la forma de libros y artículos científicos y la técnica a utilizar es el método historiográfico.

Por último, la selección de los autores se realiza de forma tal de abarcar el abanico de concepciones que se encuentran en discusión, en torno a cómo explicar el desempeño del sector agrario pampeano en el período mencionado (desde mediados de los años noventa hasta fines de la primera década de los años dos mil). Luego de una extensa revisión bibliográfica, se seleccionan los autores -y los textos- más representativos de cada una de las corrientes identificadas, sobre los cuales se centra la presente investigación. En este sentido, no se pretende incluir de manera exhaustiva los distintos autores, sino a aquellos que, a nuestro entender, representen de la mejor manera las distintas concepciones que se pretenden caracterizar en función de los objetivos del presente trabajo.

El desarrollo del presente trabajo consta de tres apartados. En el primero de ellos, se presenta el contexto histórico en el cual se pretende caracterizar la situación del sector agrario pampeano desde mediados de la década del noventa. Para ello, se incluyen referencias tanto a la situación internacional como local. En el segundo apartado, se esquematizan los autores

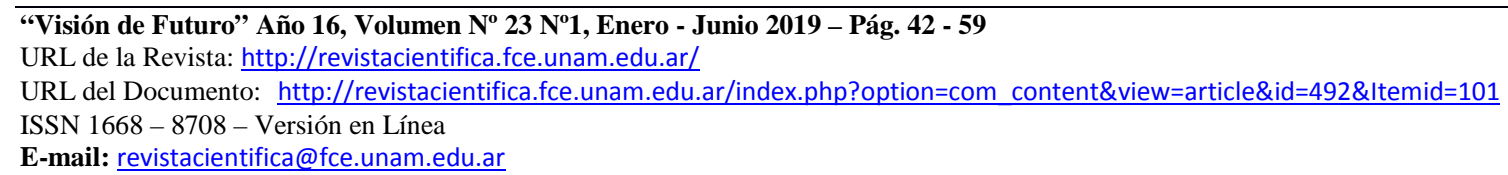


que constituyen nuestro objeto de análisis. Finalmente, en el último apartado se presentan los resultados de la investigación.

\section{DESARROLLO}

\section{El contexto histórico}

Antes de presentar el análisis de las diferentes concepciones, desarrollamos brevemente el contexto histórico en el que escriben los autores que van a ser estudiados para luego, sirviéndonos de él, poder identificar los diversos enfoques que intentan explicar el comportamiento del sector agrario de la Pampa Húmeda desde mediados de la década del noventa. Así, lo que sigue resulta una elaboración propia basada en autores que relatan la historia del agro pampeano, tales como Iñigo Carrera (1999), Barsky y Gelman (2001), Anlló et al., (2013) y Basualdo (2013).

Los últimos años del gobierno de Alfonsín, caracterizados por la gran inestabilidad de las variables macroeconómicas, coinciden con la interrupción del proceso expansivo de la agricultura pampeana (SIIA, 2017). Con el inicio del gobierno menemista, las reformas llevadas a cabo implican, para el sector agropecuario, un plan de eliminación de los derechos de exportación y la liberalización del comercio exterior, lo cual, no obstante, el sector se ve afectado por la baja de la rentabilidad y la reducción de la producción, dado que las políticas locales parecen no alcanzar para contrarrestar la caída de los precios y la demanda internacional (DataBank, 2017). Desde 1991, la profundización de la desregulación económica implica la disolución del conjunto de los organismos que actúan en el sector, la eliminación de los impuestos y tasas sobre las exportaciones, la reducción de los aranceles a la importación de insumos y productos agropecuarios y la rebaja a los impuestos de insumos como neumáticos y gasoil. Asimismo, el conjunto de las medidas macroeconómicas restablece el volumen de crédito, incluido el destinado al sector agropecuario.

Durante la década de 1990, se registran en la producción agraria pampeana efectos diversos. Por un lado, la intensificación de las inversiones de capital, la profundización de los cambios tecnológicos y la expansión de la frontera agropecuaria iniciados en los años previos producen procesos expansivos. Las medidas económicas del gobierno menemista logran que la rentabilidad del sector mejore lo cual se potencia con la gran suba de los precios internacionales de los cereales y las oleaginosas que tiene lugar hacia mediados de la década, entre 1994 y 1997. En la campaña 1997/98, Argentina se convierte en el segundo exportador mundial de harina de soja y en el primer exportador de aceite de soja y de aceite y pellets de girasol (DataBank, 2017).

\footnotetext{
"Visión de Futuro" Año 16, Volumen N²3 N¹, Enero - Junio 2019 - Pág. 42 - 59

URL de la Revista: http://revistacientifica.fce.unam.edu.ar/

URL del Documento: http://revistacientifica.fce.unam.edu.ar/index.php?option=com content\&view=article\&id=492\&ltemid=101 
En relación a la profundización del cambio tecnológico, los procesos de liberalización de los mercados posibilitan la reducción de los precios de los insumos y maquinarias agrícolas. Así, la mejora de los precios internacionales hacia mediados de la década de 1990 (DataBank, 2017), junto con la recuperación del crédito, inducen al crecimiento de las inversiones en el sector. Los pools de siembra se constituyen en una herramienta por la cual se canaliza el ahorro proveniente de otros sectores hacia el agropecuario. Los mismos cuentan con la ventaja de producir a mayor escala, logrando mejores condiciones de comercialización tanto para la compra de insumos como para la venta de sus productos. Asimismo, la diversificación de la producción que los caracteriza permite la reducción de los riesgos climáticos. También consolidan el cambio tecnológico al utilizar mayor cantidad de insumos y al contar con el asesoramiento de profesionales. Asimismo, su accionar contribuye a la concentración de la producción, aunque no modifica la propiedad de las explotaciones. Los avances que se incorporan en estos años tienen que ver con dispositivos de precisión, sensores, comandos electrónicos, sistemas de posicionamiento geográfico satelital y el riego complementario para la agricultura extensiva. A su vez, se multiplica el uso de herbicidas, fungicidas, insecticidas y fertilizantes.

No obstante, la innovación más decisiva de la década es la introducción de variedades transgénicas de soja y maíz, las cuales implican la aplicación de un paquete tecnológico nuevo integrado fundamentalmente por la mayor difusión de la siembra directa, la incorporación de maquinaria más compleja y el aumento del uso de fertilizantes y agroquímicos -especialmente glifosato (Trigo, 2016). El nuevo paquete implica menores costos -debido a la reducción de los trabajos de labranza-, un mejor control de malezas y aumentos de los rendimientos. La soja transgénica no sólo tiene la particularidad de ser resistente a los herbicidas (glifosato) y de poder ser introducida en zonas que hasta entonces son no cultivables, sino que también tiene la propiedad de ser autógama, lo cual implica que es una semilla autoreproducible. Esta característica -la cual no poseen las semillas híbridas- facilita su rápida difusión.

Hacia fines de la década de 1990 se produce una importante caída de los precios internacionales, la cual repercute en la rentabilidad de los productores (DataBank, 2017). En este período también se observa la desaparición de una cantidad importante de explotaciones (SIIA, 2017).

Finalizada la convertibilidad, la devaluación del año 2001 implica una gran transferencia de riqueza hacia los sectores exportadores, entre ellos el agropecuario. Si bien se restablecen los derechos de exportación, los mismos no llegan a contrarrestar los efectos de la devaluación. Asimismo, gran parte de los productores agropecuarios que se habían endeudado fuertemente durante la década anterior, gracias a la pesificación asimétrica, licúan sus deudas.

\footnotetext{
"Visión de Futuro" Año 16, Volumen No 23 Nº1, Enero - Junio 2019 - Pág. 42 - 59

URL de la Revista: http://revistacientifica.fce.unam.edu.ar/

URL del Documento: http://revistacientifica.fce.unam.edu.ar/index.php?option=com_content\&view=article\&id=492\&ltemid=101

ISSN 1668 - 8708 - Versión en Línea

E-mail: revistacientifica@fce.unam.edu.ar
} 
A partir de allí, comienza un período favorable para el sector: se expande el mercado interno y se recuperan los precios internacionales (DataBank, 2017). Asimismo, desde el Estado se incrementan los recursos al INTA (Instituto Nacional de Tecnología Agropecuaria) y al desarrollo de ciencia y técnica para el sector.

Comienza entonces en la década del 2000 un proceso de expansión de la producción, el cual se refleja tanto en las mejoras de los rendimientos como en la extensión de la frontera agrícola (SIIA, 2017). Con respecto a esto último, por un lado, los cambios tecnológicos permiten el cultivo en zonas no tradicionales, especialmente la siembra directa permite trabajar con menores niveles de humedad; por el otro, las variedades transgénicas permiten la realización de dos cosechas anuales, lo cual significa una duplicación del área utilizable (Lacelly e Ybran, 2016).

En el proceso de expansión agrícola de la primera década de los 2000 es decisivo el papel de la soja (Leavy y Allen, 2015). Desde su introducción en la década de 1970, llega a representar más de la mitad de la superficie sembrada y del volumen producido a mediados de la década del 2000 (SIIA, 2017).

Por último, en relación a las innovaciones, en los años recientes se avanza en la introducción de la 'agricultura de precisión'. Así, cada vez más, la incorporación de las complejidades tecnológicas implica que las producciones requieran un uso más intensivo de capital y una escala mínima de explotación mayor, así como un proceso de gestión más complejo, todo lo cual induce a la concentración de la producción. En relación a los actores protagonistas de la profundización del cambio tecnológico que ocurre durante la posconvertibilidad, puede apreciarse el rol creciente que tienen los contratistas de maquinaria y los proveedores de insumos.

En conclusión, desde comienzos del nuevo siglo, la consolidación del nuevo paquete productivo, basado en la soja, ocurre al tiempo que Argentina, año tras año, logra récords de producción y aumenta sus participaciones en los mercados internacionales (DataBank, 2017). Desde mediados de la década de 1990, la producción agraria pampeana se duplica con creces, como resultado no sólo del aumento de la superficie sembrada, sino también de los rendimientos (SIIA, 2017). Se reducen las brechas productivas y en los cultivos pampeanos los rindes argentinos se asemejan a los registros internacionales. Sin dudas, esta expansión agrícola nacional se da al mismo tiempo que la demanda internacional -fundamentalmente, de origen chino- crece sostenidamente (Oviedo, 2015; Desai, 2015).

\section{Las explicaciones económicas sobre el agro pampeano}

Para la presentación de las concepciones que surgen a partir del boom de la producción sojera que comienza a consolidarse a mediados de la década de 1990, se proponen

\footnotetext{
“Visión de Futuro" Año 16, Volumen No 23 N¹, Enero - Junio 2019 - Pág. 42 - 59

URL de la Revista: http://revistacientifica.fce.unam.edu.ar/

URL del Documento: http://revistacientifica.fce.unam.edu.ar/index.php?option=com content\&view=article\&id=492\&ltemid=101 
subgrupos que pretenden dar cuenta del motor explicativo encontrado en cada uno de los autores. En relación a este punto, es importante señalar que la división de las explicaciones en conjuntos no implica que las mismas no tengan puntos de contacto entre ellas. En consecuencia, lo que guía la estructuración propuesta es la identificación, en cada uno de los casos, del motor explicativo que, entendemos, estructura todo el argumento de los autores, lo cual no invalida que, sin perjuicio de ello, pueda haber otros determinantes en las explicaciones.

Adicionalmente, la propuesta que sigue no pretende ser exhaustiva. Por el contrario, el objetivo es analizar la literatura especializada incluyendo una variedad significativa de autores de modo tal de poder verificar las hipótesis del presente trabajo.

Desde mediados de la década de 1990, los récords en los niveles de la producción agraria pampeana vienen acompañados por transformaciones productivas y tecnológicas significativas, las cuales, por lo tanto, conllevan, a su vez, aumentos en los rendimientos por hectárea. En cada uno de los casos, los autores rescatan un aspecto que caracteriza el desempeño del sector y lo convierten en el motor de ese comportamiento. Así, están quienes ponen el foco en la dinámica propia de los paradigmas tecnológicos (1.), aquellos que lo hacen en el contexto favorable, ya sea internacional, entendiendo al proceso como de índole global, o ya sea local, gracias a la política económica argentina (2.), quienes consideran que lo fundamental es la presencia de nuevos actores -los pools de siembra- (3.) y los que estructuran su explicación analizando las relaciones de poder -en este caso, lo determinante es que la oligarquía vuelve a formar parte del bloque dominante- (4.).

Entendemos que en cada concepción puede extraerse una característica saliente del proceso bajo análisis, a partir de la cual se construye el argumento, sin dejar de lado otros rasgos que aparecen como contextuales o complementarios.

\section{Dinámica del proceso de innovación: cambio de paradigma tecnológico}

Anlló et al., (2013) sostienen que el extraordinario desempeño del sector en los años 2000 se debe a un proceso de cambio de paradigma tecnológico, organizacional y productivo, el cual se enmarca en la revolución biológica en curso a nivel mundial. Esta explicación se inscribe en la teoría evolucionista, la cual entiende que los sistemas económicos son dinámicos y evolucionan a lo largo del tiempo, a través de procesos de aprendizaje, interacción con el entorno e influencia del pasado. Por lo tanto, desde esta perspectiva, los ciclos económicos se explican a partir de la sucesión de paradigmas tecnológicos. La consolidación de un nuevo paradigma explica la fase ascendente del ciclo, y el agotamiento de un viejo paradigma, la fase descendente. El cambio de un paradigma por otro se inicia,

\footnotetext{
“Visión de Futuro" Año 16, Volumen No 23 N¹, Enero - Junio 2019 - Pág. 42 - 59

URL de la Revista: http://revistacientifica.fce.unam.edu.ar/

URL del Documento: http://revistacientifica.fce.unam.edu.ar/index.php?option=com_content\&view=article\&id=492\&ltemid=101

ISSN 1668 - 8708 - Versión en Línea

E-mail: revistacientifica@fce.unam.edu.ar
} 
para estos autores, cuando un conjunto de renovados empresarios se arriesga a implementar nuevas tecnologías, logrando así captar rentas.

Específicamente en relación al desempeño del sector desde mediados de la década de 1990, estos autores explican que lo que se produce es la consolidación de un nuevo paradigma, cuyos primeros indicios comienzan a producirse en la década de 1970. A su entender, el agotamiento del sistema previo induce diversas respuestas que van sentando las bases de las tecnologías que luego serían básicas en el nuevo modelo (mayor mecanizaciónespecialmente tractores-, híbridos, primeros avances en siembra directa, uso más intensivo de biocidas y fertilizantes). No obstante, es en la década de 1990 que el incipiente dinamismo de los mercados internacionales, más las reformas en la economía local -con su correspondiente reflejo en los precios relativos- producen las condiciones para que se lleve a cabo un abrupto proceso de capitalización caracterizado por modernización tecnológica, concentración productiva y creciente endeudamiento. Luego, continúan los autores, con la salida de la convertibilidad, finalmente se da la consolidación del nuevo modelo productivo y tecnológico que mejora notablemente la rentabilidad del sector. De esta forma, luego de varias décadas, el agro argentino vuelve a evidenciar un dinamismo similar al que lo caracterizó a principios del siglo XX.

Por último, cabe mencionar que también el contexto, no obstante, juega un rol importante para los autores Bisang et al. (2015). Para ellos, recién iniciada la década de 1970, comienzan a tener lugar las primeras precondiciones para los futuros cambios, debido a la modificación de la política económica. Sin embargo, es a partir de la década de 1990 que se produce la consolidación del cambio por la conjunción de varios factores, a saber: el desmantelamiento de una serie de regulaciones económicas locales, la recuperación de los precios internacionales y la consolidación de nuevos cultivos (soja). Así, en esos años, se produce un proceso de crecimiento con endeudamiento apelando al cambio tecnológico. En la segunda mitad de la década de 1990, los autores explican que la conjunción del endeudamiento del sector con las condiciones internacionales desfavorables y los problemas de la convertibilidad, inducen a la incorporación masiva de técnicas y tecnologías ahorradoras de costos y de un nuevo modelo organizacional (agricultura bajo contrato). Con el fin de la convertibilidad, se produce la pesificación asimétrica (se licúan los pasivos del sector), mejora la rentabilidad y aparece la posibilidad de autofinanciamiento por parte del sector. Luego, las capacidades tecnológicas acumuladas durante la década anterior, las reducciones en los pasivos, las mejoras en los precios internacionales y, más adelante, en los precios relativos internos, conducen a los nuevos récords productivos, continúan Anlló et al. Todo esto, claro, acompañado -y posibilitado- por un aumento de la demanda internacional. Más tarde, en la segunda mitad de la primera década del siglo XXI, los precios internacionales explotan, lo cual

\footnotetext{
“Visión de Futuro" Año 16, Volumen No 23 N¹, Enero - Junio 2019 - Pág. 42 - 59

URL de la Revista: http://revistacientifica.fce.unam.edu.ar/

URL del Documento: http://revistacientifica.fce.unam.edu.ar/index.php?option=com content\&view=article\&id=492\&ltemid=101 
constituye un nuevo impulso para el crecimiento de la producción, el aumento de la frontera y la profundización del modelo tecnológico, concluyen los autores. En síntesis, desde este enfoque, el extraordinario desempeño del agro pampeano en los años 2000 es la consecuencia de la consolidación del nuevo paradigma tecnológico, siendo que la sucesión de paradigmas responde a una lógica propia, aunque haya contextos más favorables para su afianzamiento (tanto en términos locales como internacionales).

\section{Contexto favorable}

Pierri y Abramovsky (2010) también hacen hincapié en la adopción (y consolidación) del nuevo paquete tecnológico para explicar el boom de la soja y los récords productivos de las últimas décadas, lo cual, señalan, es posibilitado por la mejora de la rentabilidad que se produce gracias a la creciente demanda de los países de Oriente y al cambio en la política económica -fundamentalmente, la devaluación que implica que se licúen los pasivos de un sector fuertemente endeudado. En otros trabajos (Pierri, 2004; Pierri y Junior, 2017), no obstante, estos autores advierten que la literatura suele sobrevalorar la importancia de la innovación tecnológica y el esfuerzo privado, minimizando el rol del Estado y el contexto mundial- en cuanto a las posibilidades de que la producción encuentre demanda internacional. De esta manera, buscan destacar, por un lado, la influencia de las iniciativas estatales para mejorar la producción y la exportación de soja y, por el otro, el contexto internacional en el cual la producción -y la demanda- de soja explota. Así, la conclusión principal de este enfoque es que las explicaciones sobre el desempeño del sector no deben centrarse exclusivamente en aspectos locales, siendo fundamental el contexto internacional, esto es, la extraordinaria demanda mundial para la producción agraria pampeana, la cual repercute en los precios de dichos productos.

También resaltando la importancia de entender el fenómeno a nivel mundial, Anino y Mercatante (2009) sostienen que los aumentos de rendimientos de las últimas décadas se deben a procesos globales de avances tecnológicos y ampliación de la frontera agropecuaria en todo el mundo. No obstante, los autores reconocen que Argentina se beneficia relativamente más que otros países de las mejoras que permiten aumentar los rendimientos en el agro. A su entender, lo que diferencia el proceso argentino y explica el aumento de la renta, es la escala masiva con la cual se aplican las nuevas tecnologías que simplifican el proceso productivo. En nuestro país, desde la década de 1970 se inicia un proceso de agriculturización que va aumentando los rendimientos por hectárea y la rentabilidad de los cultivos. La introducción de la semilla transgénica posibilita el desarrollo de procesos productivos más sencillos y estandarizados que descansan mucho más en la maquinaria y menos en el gasto de fuerza de trabajo. Así, explican los autores, la reducción del trabajo

“Visión de Futuro” Año 16, Volumen No 23 N¹, Enero - Junio 2019 - Pág. 42 - 59

URL de la Revista: http://revistacientifica.fce.unam.edu.ar/

URL del Documento: http://revistacientifica.fce.unam.edu.ar/index.php?option=com content\&view=article\&id=492\&ltemid=101

ISSN 1668 - 8708 - Versión en Línea

E-mail: revistacientifica@fce.unam.edu.ar 
necesario redunda en un aumento de la productividad (mismos rindes con menos trabajo), lo cual genera más renta y ganancias extraordinarias. La conclusión de los autores es entonces que el aumento de los rindes y el corrimiento de la frontera que se producen en el ámbito local son parte de un proceso global orquestado por las semilleras multinacionales, los proveedores de agroquímicos y las exportadoras de granos. Así, la modernización del agro pampeano se da a la vez que se profundiza su carácter subordinado. A entender de los autores, el proceso que se refleja en el aumento de la producción agrava, no obstante, la inserción dependiente de Argentina, como apéndice agrario de los países centrales.

Barsky y Gelman (2001), al analizar el desempeño del sector desde las últimas décadas del siglo XX, señalan que, en la década de 1990, se produce una nueva expansión productiva la cual, si bien se basa en la profundización del cambio tecnológico, no hubiera podido darse sin la extraordinaria suba de los precios internacionales de los cereales y las oleaginosas. A mediados de la década de 1990 se supera la cosecha récord de la década anterior, lo cual identifican como continuación de lo que ya viene ocurriendo en el sector, en relación a la expansión productiva, la profundización del cambio tecnológico y la ampliación de la frontera agrícola.

No obstante, los autores señalan que, en los últimos años del siglo XX, el sector se ve perjudicado al enfrentar condiciones adversas: fundamentalmente, los precios internacionales descienden a sus niveles anteriores (habían alcanzado valores muy altos entre 1995 y 1997), lo cual se conjuga con un contexto local de inestabilidad política y económica (suba de la tasa de interés y de los precios internos). Esta situación se revierte, continúan Barsky y Gelman, en los 2000, gracias a que el cambio de la política económica, sumado a las posibilidades dadas por la consolidación del cambio tecnológico (tanto el aumento de los rendimientos como la expansión de la frontera agrícola), provoca saltos espectaculares en la producción de cereales y oleaginosas. De esta manera, vemos que esta perspectiva, al explicar la evolución del desempeño del sector, prioriza el contexto internacional, específicamente los precios internacionales, destacando, en segundo lugar, la influencia de las políticas locales.

Del mismo modo que Pierri, et al., (2006) también destacan el rol del Estado para explicar el proceso que atraviesa el agro pampeano en las últimas décadas, aunque en este caso aparece como el factor determinante en el extraordinario desempeño del sector. Estos autores explican que, a partir de la devaluación, se produce un sinceramiento de la estructura de precios relativos, que implica una reducción de los costos en dólares para el sector y conlleva, en consecuencia, una multiplicación de la rentabilidad. Así, la conclusión de Rodríguez y Arceo (2006) es que el extraordinario crecimiento de la producción agraria de la Pampa Húmeda se produce por el incremento de la rentabilidad que tiene lugar a partir del fin de la convertibilidad. En este sentido, para estos autores, el rol del Estado es clave para

\footnotetext{
"Visión de Futuro" Año 16, Volumen N²3 N¹, Enero - Junio 2019 - Pág. 42 - 59

URL de la Revista: http://revistacientifica.fce.unam.edu.ar/

URL del Documento: http://revistacientifica.fce.unam.edu.ar/index.php?option=com content\&view=article\&id=492\&ltemid=101

ISSN 1668 - 8708 - Versión en Línea

E-mail: revistacientifica@fce.unam.edu.ar
} 
explicar el desempeño del agro pampeano de principios de siglo XXI, dado que la causa última es la política económica que se lleva a cabo.

\section{Nuevos actores}

Mirando el desempeño del sector en los últimos años, Fernández (2010) señala que se desarrolla una nueva forma de producción, liderada por los pools de siembra. A su entender, el crecimiento de la producción es consecuencia del ahorro de capital que deriva del hecho de que la producción se realice a mayor escala, con la correspondiente homogeneización de la tecnología; pues, señala Fernández, el proceso liderado por los pools de siembra permite la liberación de capital que puede utilizarse de otras maneras (como, por ejemplo, ofrecerse como renta para el alquiler de más tierras para ampliar la escala), aunque no implica que se produzca con mayores rindes por hectárea. Esto no conduce necesariamente a mayor inversión sino que a inversión más potente -menores costos por hectárea sembrada- por el aprovechamiento de las economías de escala. En este sentido, esta explicación hace hincapié en el rol de los pools de siembra -esto es, la aparición de nuevos actores en el sector- para entender el boom productivo del agro pampeano. A su entender, los pools de siembra implican que se produzca de manera más eficiente, lo cual conduce al crecimiento de la producción.

\section{Relaciones de poder}

Basualdo (2013) también identifica que el extraordinario desempeño del sector se relaciona con el surgimiento y la consolidación de un nuevo paradigma y se propone investigar los procesos y actores que hay detrás.

A entender de este autor, si bien los paradigmas productivos y tecnológicos se desarrollan internacionalmente, su incorporación en Argentina depende de la decisión de los propietarios de la tierra, quienes los evalúan en función de su maximización de beneficios. Así, lo ocurrido en la década de 1990, cuando madura un nuevo paradigma agropecuario, no es la excepción en este sentido: el propietario es el actor fundamental en el modelo sojero. En consecuencia, esta perspectiva cuestiona la idea de la consolidación de un nuevo tipo de propietario innovador, dado que sostiene que las transformaciones productivas y tecnológicas que tienen lugar en el último cuarto del siglo $X X$ son llevadas a cabo por los grandes terratenientes tradicionales, a partir de la interrupción de la sustitución de importaciones en 1976 (Arceo y Basualdo, 2005).

Para entender la transformación del agro pampeano, desde esta perspectiva, debe comprenderse que a partir del gobierno de facto que se instala en marzo de 1976, se inicia un proceso conducido por la oligarquía -que se constituye nuevamente en el bloque dominante, el cual significa una interrupción forzada de la industrialización sustitutiva. Como "Visión de Futuro" Año 16, Volumen No 23 N¹, Enero - Junio 2019 - Pág. 42 - 59 URL de la Revista: http://revistacientifica.fce.unam.edu.ar/

URL del Documento: http://revistacientifica.fce.unam.edu.ar/index.php?option=com content\&view=article\&id=492\&ltemid=101 ISSN 1668 - 8708 - Versión en Línea

E-mail: revistacientifica@fce.unam.edu.ar 
consecuencia, se produce una alteración de los precios relativos en favor de la oligarquía pampeana, lo cual redunda en un incremento de la producción y la productividad del sector agropecuario. Finalizada la dictadura, con los primeros gobiernos democráticos, especialmente durante la convertibilidad, se consolidan las tendencias hacia la desindustrialización, provocando el tránsito de una economía industrial a otra financiera, agropecuaria y de servicios, señala el autor.

En este contexto, en la explicación de Basualdo, el cambio tecnológico es llevado a cabo por el sujeto histórico del sector, esto es, el propietario. Así, el actual paradigma sojero no sólo no cuestiona la importancia del propietario, sino que es el actor central en su funcionamiento, aunque adquiera nuevas características. Al exacerbarse las economías de escala, se potencia la importancia de la gran propiedad, por lo que la centralidad de la producción agropecuaria es ejercida por una fracción específica de los propietarios: los terratenientes pampeanos. En síntesis, en este planteo, la transformación del agro pampeano se explica, fundamentalmente, por el rol central de la oligarquía dentro del bloque dominante a partir de 1976. Así, los terratenientes pampeanos desde su posición dominante deciden la introducción de un nuevo paradigma productivo y tecnológico -el cual se encuentra disponible internacionalmente- porque les significa mayores ingresos.

\section{El análisis de las explicaciones económicas sobre el agro pampeano: el método hipotético-deductivo popperiano}

El proceso de conocimiento de la ciencia moderna, desde la perspectiva del método hipotético-deductivo popperiano (Popper, 1962), consiste en la búsqueda de hipótesis que puedan ser evaluadas en función de su correspondencia con la realidad, esto es, que puedan ser contrastadas con la evidencia empírica a ser recolectada en relación a determinado fenómeno que se quiere conocer.

El problema al que se enfrenta Popper es evidenciar el desarrollo del conocimiento científico con principios objetivos, dado que, a su entender, no es posible justificar, desde la lógica y la razón, la conexión desde los enunciados singulares a un enunciado universal. En otras palabras, el autor se enfrenta a la problemática que implica el método inductivo en relación a cómo justificar los enunciados universales. Así, en su intento de justificar el proceso de conocimiento científico, señala que el funcionamiento de la ciencia consiste en proponer las hipótesis acerca de una determinada porción de la realidad -el fenómeno que se pretender explicar-, a partir de las cuales, a través de la deducción, se puedan obtener conclusiones que tengan la forma de predicciones contrastables, esto es, que puedan compararse con la base empírica, la cual está constituida por un conjunto de enunciados singulares cuya veracidad no es puesta en duda.

\footnotetext{
"Visión de Futuro" Año 16, Volumen No 23 N¹, Enero - Junio 2019 - Pág. 42 - 59

URL de la Revista: http://revistacientifica.fce.unam.edu.ar/

URL del Documento: http://revistacientifica.fce.unam.edu.ar/index.php?option=com content\&view=article\&id=492\&ltemid=101 
Específicamente, las predicciones deben ser enunciados que puedan ser falsados a partir de la empiria; pues, para Popper, si bien es posible concluir la falsedad de una hipótesis, en cambio, no puede concluirse su verdad. De esta manera, el proceso de conocimiento consiste en someter a prueba de manera reiterada la capacidad de las hipótesis de no ser rechazadas por no encontrarse, hasta el momento, experiencia empírica que contradiga las predicciones que se desprenden de ellas. En caso de que una predicción sea contradictoria con la experiencia empírica, la hipótesis de la cual se deriva ha sido falsada. La falsación de una hipótesis significa que la misma no se adecúa a la experiencia práctica, siendo que esta última es considerada un fiel reflejo del fenómeno que se pretende explicar. Esta característica fundamental del método presentado por Popper es la que le da el nombre de falsacionismo. La consecuencia del método tal como lo expone el autor es que la verdad nunca puede ser alcanzada directamente: las teorías, las hipótesis, no pueden demostrarse como verdaderas. Contrariamente, éstas se conservan como corroboradas mientras que no haya un enunciado singular de la base empírica que contradiga las predicciones que de ellas se deriven.

Desde esta óptica, no resulta problemático, para el desarrollo de la ciencia, el hecho de que no pueda saberse si se ha alcanzado la verdad, debido a que el método garantiza ir acercándose a ella; pues, la ciencia avanza a través de las refutaciones, en un camino en el cual la verdad absoluta actúa como principio regulador. De esto se deriva la importancia de que los sistemas teóricos sean susceptibles de ser falsados para que puedan ser parte de la ciencia. A su vez, este método sostiene que la verdad no sólo es estática, sino que inmediata, dado que las hipótesis se contrastan con enunciados singulares descriptivos (base empírica). En consecuencia, desde esta concepción, al pretender explicar la realidad es formalmente posible abordarla captando aspectos parciales de los fenómenos.

A continuación, nos proponemos ver cómo esta forma de conocimiento se presenta en las concepciones que pretenden explicar el desempeño agrario pampeano. Como adelantamos en la introducción, el objetivo del presente trabajo es, una vez identificado el recurrente disenso que se verifica en la literatura sobre el desempeño agrario pampeano reciente, estudiar las distintas concepciones a través de un análisis metodológico. La hipótesis que guía dicho análisis es que la falta de consenso entre los autores proviene de la forma general de abordaje de los fenómenos. En este sentido, el método popperiano describe el proceso de elaboración de hipótesis (o teorías) y, por lo tanto, como se intenta poner de manifiesto a continuación, aquel que, entendemos, se vincula directamente con la existencia de una multiplicidad de concepciones igualmente válidas (esto es, la falta de consenso).

En el apartado precedente, presentamos una variedad de explicaciones referidas al desempeño del sector agrario pampeano reciente. En función de la estructuración propuesta, pretendemos poner de manifiesto cómo, más allá de la observación de los mismos "Visión de Futuro" Año 16, Volumen No 23 N¹, Enero - Junio 2019 - Pág. 42 - 59 URL de la Revista: http://revistacientifica.fce.unam.edu.ar/

URL del Documento: http://revistacientifica.fce.unam.edu.ar/index.php?option=com content\&view=article\&id=492\&ltemid=101 ISSN 1668 - 8708 - Versión en Línea

E-mail: revistacientifica@fce.unam.edu.ar 
fenómenos, se construyen explicaciones que distan unas de otras. En otras palabras, las distintas concepciones, incluso con una misma caracterización de aquello de lo que pretenden dar cuenta, argumentan sobre el desempeño de la producción agraria pampeana identificando distintos motores explicativos.

Así, frente al fenómeno que se pretende explicar -el extraordinario desempeño de los 2000-, los distintos enfoques parten de identificar los elementos que consideran relevantes. De esta manera, en casi todas las concepciones, aparecen los mismos elementos característicos: la transformación del proceso productivo, el cambio tecnológico, la mejora de los precios internacionales y la modificación de la política económica local que se vuelve más favorable para el sector (apertura y liberalización económica desde la década de 1990 y devaluación en 2002). Nuevamente, no sólo cada enfoque destaca distintos elementos de ese conjunto, sino que luego se estructuran diferentes explicaciones partiendo incluso desde los mismos postulados.

La forma en la cual presentamos los enfoques -identificando el motor explicativo de cada concepción- pretende mostrar esta característica. En línea con este propósito también, desarrollamos el contexto histórico en el cual escriben los distintos autores con el objetivo de poner de manifiesto que, aun argumentando de manera distinta a la hora de dar cuenta sobre el desempeño del sector, puede encontrarse una caracterización común del momento que analizan. En otras palabras, al recorrer la literatura especializada pueden identificarse, en la multiplicidad de enfoques, elementos comunes para caracterizar el fenómeno a explicar, los cuales pretendemos resaltar al agruparlos en el apartado del contexto histórico.

Luego, clasificamos los autores en función del motor explicativo. Nuevamente, esta forma de estructurar el apartado pretende dar cuenta que, luego de la selección de los elementos relevantes, en cada caso hay una hipótesis distinta para explicar lo que efectivamente ocurre, lo cual no pareciera tener otro origen que la subjetividad de cada autor.

\section{CONCLUSIÓN}

El presente trabajo se ha desarrollado en dos partes complementarias e intrínsecamente vinculadas. La primera de ellas referida al estudio de la literatura sobre el desempeño agrario pampeano reciente, y la segunda a su análisis metodológico.

De esta manera, como punto de partida de la primera parte de esta investigación, hemos realizado una revisión de la literatura especializada que aborda el desempeño de la producción agraria pampeana. El resultado de dicho estudio nos ha llevado a corroborar el disenso entre los autores para explicar el comportamiento reciente del sector. De esta forma, se ha satisfecho el objetivo específico de esta investigación que ha consistido en verificar que

\footnotetext{
“Visión de Futuro" Año 16, Volumen N 23 N¹, Enero - Junio 2019 - Pág. 42 - 59

URL de la Revista: http://revistacientifica.fce.unam.edu.ar/

URL del Documento: http://revistacientifica.fce.unam.edu.ar/index.php?option=com content\&view=article\&id=492\&ltemid=101

ISSN 1668 - 8708 - Versión en Línea

E-mail: revistacientifica@fce.unam.edu.ar
} 
efectivamente está produciéndose nuevamente una época de disenso entre los estudiosos de la producción agraria pampeana, en relación al desempeño del sector en los años 2000.

El estudio de la literatura especializada se ha podido llevar a cabo en pos de la búsqueda del motor explicativo esgrimido en cada uno de los casos. De esta forma, el resultado ha sido una estructuración que implica una clasificación de los diversos enfoques en función de cuál es la causa principal del extraordinario desempeño del sector, en línea con las hipótesis del trabajo.

A continuación, en la segunda parte del trabajo, en el análisis metodológico de las explicaciones sobre el agro de la Pampa Húmeda, se ha identificado la correspondencia entre la forma de abordaje de las concepciones revisadas en la primera parte del trabajo y el método hipotético-deductivo popperiano, sustento epistemológico de la forma de conocer de la ciencia económica convencional.

La identificación de este pilar epistemológico como sustento de las concepciones sobre el agro pampeano estudiadas previamente, nos ha permitido la corroboración de la hipótesis según la cual la falta de consenso entre los autores proviene de la forma general de abordaje de los fenómenos en la ciencia económica. En otras palabras, hemos identificado cómo la multiplicidad de enfoques se deriva de que cada uno de ellos, luego de seleccionar los elementos que considera relevantes para la explicación a elaborar, hipotetiza acerca del comportamiento de dichos elementos, en concordancia al método hipotético-deductivo. Así, cada concepción, primero realiza un recorte específico de la realidad y luego, elabora hipótesis para, relacionando las características seleccionadas, construir el argumento que dé cuenta del fenómeno que se pretende explicar.

De esta forma, la reflexión metodológica de los autores nos ha permitido echar luz sobre las potencialidades y limitaciones de cada uno de ellos para comprender el sector agrario pampeano. En línea con esto, la vinculación entre el método popperiano y la multiplicidad de enfoques (igualmente válidos) para dar cuenta de los mismos fenómenos observados, ha mostrado la necesidad de cuestionar la forma de abordaje de aquello de lo que se pretende dar cuenta por parte de los análisis económicos.

Así, la presente investigación, que se ha sustentado en la necesidad de una mirada complementaria acerca del desempeño y las características de la producción agraria pampeana tanto para comprenderla en la unidad de la acumulación de capital en Argentina y su papel en la División Internacional del Trabajo (DIT), como para contar con mayores herramientas a la hora de planificar estrategias de producción en el agro pampeano, ha señalado como punto de partida el imperativo de reflexionar críticamente sobre el modo de abordaje de los fenómenos. En otras palabras, hemos encontrado que el estudio del disenso en la literatura económica agraria, haciendo hincapié en la cuestión metodológica en la cual "Visión de Futuro" Año 16, Volumen No 23 Nº1, Enero - Junio 2019 - Pág. 42 - 59 URL de la Revista: http://revistacientifica.fce.unam.edu.ar/

URL del Documento: http://revistacientifica.fce.unam.edu.ar/index.php?option=com content\&view=article\&id=492\&ltemid=101 ISSN 1668 - 8708 - Versión en Línea

E-mail: revistacientifica@fce.unam.edu.ar 
se sustenta, se presenta como caso particular de la crítica del método de conocimiento científico, lo cual abre la perspectiva de ahondar en el estudio de sus potencialidades y limitaciones, con el objeto de avanzar hacia su superación.

\section{REFERENCIAS}

Anino, P. y Mercatante, E., (2009). Renta agraria y desarrollo capitalista en Argentina. Lucha de Clases. Número 9, 69-110.

Anlló, G., Bisang, R., y Campi, M. (2013). Claves para repensar el agro argentino. Buenos Aires, Argentina. Eudeba.

Arceo, N. y Basualdo, E., (2005). Incidencia y características productivas de los grandes terratenientes bonaerenses durante la vigencia del régimen de convertibilidad. Desarrollo Económico, Volumen 45, (Número 177), 75-97.

Barsky, O. y Gelman, J. (2001). Historia del agro argentino. Desde la conquista hasta fines del siglo XX. Buenos Aires, Argentina. Mondadori.

Basualdo, E. (2013). Estudios de historia económica argentina. Desde mediados del siglo XX a la actualidad. Buenos Aires, Argentina. Siglo XXI.

Bisang, R., Anlló, G., y Campi, M. (2015). Políticas tecnológicas para la innovación: la producción agrícola argentina. Innovación tecnológica. CIEPLAN. Recuperado de: http://scioteca.caf.com/handle/123456789/773

DataBank (2017). Base de Datos del Banco Mundial. Recuperado de: http://databank.worldbank.org/data/home.aspx

Denari, L., (1991). Economía y epistemología. Los desaciertos del conocimiento científico. Realidad Económica. (Número 103), 81-95.

Desai, A., (2015). El crecimiento de China y sus vinculaciones con la industria de soja en la Argentina / Chinese Growth and its Connections with the Argentinian Soy Industry. Independent Study Project (ISP) Collection. Recuperado de: http://digitalcollections.sit.edu/isp_collection/2116

Fernández, D., "Reflexiones sobre el crecimiento agrícola pampeano a la luz del concepto de renta diferencial de segundo tipo". Documentos del CIEA, 2010, Volumen 6, 5-29.

https://fernandezdiego.weebly.com/uploads/5/3/3/5/53355101/articulodocs6.pdf

Iñigo Carrera, J., (1999). La acumulación de capital en Argentina. CICP. Recuperado de: http://cicpint.org/wp-content/uploads/2017/03/1998_JIC_La-acumulacion-de-capital-enla-Argentina.pdf

INTA (2016). Informe estadístico del mercado de la soja. Recuperado de:

https://inta.gob.ar/sites/default/files/inta_informe_estadistico_del_mercado_de_soja.pdf.

\footnotetext{
"Visión de Futuro" Año 16, Volumen No 23 N¹, Enero - Junio 2019 - Pág. 42 - 59

URL de la Revista: http://revistacientifica.fce.unam.edu.ar/

URL del Documento: http://revistacientifica.fce.unam.edu.ar/index.php?option=com content\&view=article\&id=492\&ltemid=101

ISSN 1668 - 8708 - Versión en Línea

E-mail: revistacientifica@fce.unam.edu.ar
} 
Leavy, S. y Allen, B., Comercialización de soja en las últimas dos décadas. IX Jornadas de Estudios Agrarios y Agroindustriales Argentinos y Latinoamericanos (03-06 de noviembre de 2015, Buenos Aires).

Oviedo, E., (2015). El ascenso de China y sus efectos en la relación con Argentina. Estudios internacionales. Volumen 47, (Número 180), 67-90.

Pierri, J., El sector externo y la producción de soja en Argentina 1960/2001. Documentos del CIEA, 2004, Volumen 2. 14/05/2018: http://www.ciea.com.ar/web/wpcontent/uploads/2016/11/Doc2_10.pdf.

Pierri, J. y Abramovsky, M., "Consideraciones sobre la inserción internacional del complejo sojero: ¿una economía "sui generis" del siglo XXI?". Documentos del CIEA, 2010, Volumen 6, 31-63. 14/05/2018:

http://bibliotecadigital.econ.uba.ar/download/docuciea/docuciea_n6_02.pdf

Pierri, J. y Junior, V., "La sojización en Argentina y Brasil (1980/2014): influencia de las políticas públicas, de las empresas transnacionales y de la estructura económica dependiente". Extensão Rural, 2017, Volumen 24, Número 1, 20-41.

Popper, K. (1962), La lógica de la investigación científica. Buenos Aires, Argentina, Tecnos.

Rodríguez, J. y Arceo, N., (2006). Renta agraria y ganancias extraordinarias en Argentina.

Realidad Económica, Volumen 219, 76-98.

Sábato, H., (1987). La cuestión agraria pampeana: un debate inconcluso. Desarrollo Económico, Volumen 106, 291-301.

SIIA (2017). Datos Abiertos de Agroindustria, Ministerio de Agroindustria de la Presidencia de la Nación Argentina. Recuperado de: https://www.agroindustria.gob.ar/datosabiertos/.

ArgenBio. (2016), Veinte años de cultivos genéticamente modificados en la agricultura argentina.

Recuperado

de:

https://www.argenbio.org/adc/uploads/20GM 2016/Informe 20GM web.pdf

\section{RESUMEN BIOGRÁFICO}

\section{Estefanía Soledad Dileo}

- Instituto de Investigaciones Económicas de la Universidad de Buenos Aires.

Formación: Doctorado en Ciencias Sociales. En curso. Universidad de Buenos Aires.

Licenciatura en Economía. Facultad de Ciencias Económicas. Universidad de Buenos Aires.

Becas de Investigación: 2015 septiembre - actualidad. Beca UBACyT Doctorado. "Las transformaciones productivas desde los noventa en la producción agrícola y su impacto en la generación y distribución de la riqueza social producida anualmente por Argentina".

\footnotetext{
“Visión de Futuro" Año 16, Volumen No 23 N¹, Enero - Junio 2019 - Pág. 42 - 59

URL de la Revista: http://revistacientifica.fce.unam.edu.ar/

URL del Documento: http://revistacientifica.fce.unam.edu.ar/index.php?option=com_content\&view=article\&id=492\&ltemid=101

ISSN 1668 - 8708 - Versión en Línea

E-mail: revistacientifica@fce.unam.edu.ar
} 